\title{
Neutrinos and Big-Bang Nucleosynthesis
}

\author{
T. KAJINO ${ }^{\text {a }}$ b c and M. ORITO ${ }^{a}$

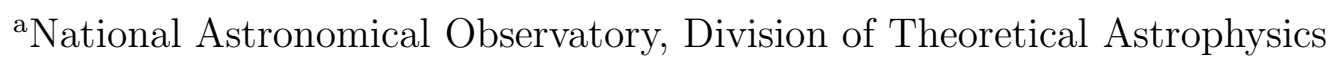 \\ ${ }^{\mathrm{b}}$ The Graduate University for Advanced Studies, Department of Astronomical Science \\ 2-21-1 Osawa, Mitaka, Tokyo 181, Japan \\ ${ }^{\mathrm{c}}$ University of Tokyo, Department of Astronomy \\ 2-11-16 Yayoi, Bunkyo-ku, Tokyo 113, Japan
}

Observations of clusters and super clusters of galaxies have indicated that the Universe is more dominated by baryons than ever estimated in the homogeneous cosmological model for primordial nucleosynthesis. Recent detections of possibly low deuterium abundance in Lyman- $\alpha$ clouds along the line of sight to high red-shift quasars have raised another potential difficulty that ${ }^{4} \mathrm{He}$ is overproduced in any cosmological models which satisfy the low deuterium abundance constraint. We show that the inhomogeneous cosmological model with degenerate electron-neutrino can resolve these two difficulties.

\section{Introduction}

One of the cosmological impacts of primordial nucleosynthesis is on the universal baryon mass density $\rho_{b}$. It is of significance to answer the question how much fraction of universal mass is made of ordinary matter baryons. Homogeneous Big-Bang model for primordial nucleosynthesis [1,2], assuming the standard model for light neutrino families, predicts small $\Omega_{b}, 0.03 \leq \Omega_{b} h_{50}^{2} \leq 0.06$, where $\Omega_{b}=\rho_{b} / \rho_{c}, \rho_{c}$ is the critical density which marginally closes the Universe, and $h_{50}$ is the Hubble constant $H_{0}$ divided by $50 \mathrm{~km} / \mathrm{s} / \mathrm{Mpc}$. However, recent observations of rich clusters and super clusters of galaxies have indicated much larger baryon fraction, $0.1 \leq \Omega_{b} h_{50}^{3 / 2} \leq 0.3$ [3, [4].

Inhomogeneous Big-Bang model [5]- 9], which allows inhomogeneous baryon density distribution due to various physical processes in the early Universe, has been proposed in order to resolve this discrepancy. In this model difference in diffusion effects between neutrons and charged nuclei plays an important role in fluctuating density distribution to suppress overproduction of ${ }^{4} \mathrm{He}$, and resultant $\Omega_{b}$ is relaxed to $\Omega_{b} \sim 0.1$ [ 8 .

However, another potential difficulty has been imposed by recent observations of deuterium absorption line in Lyman- $\alpha$ clouds along the line of sight to high red-shift quasars [10, [1]. Several detections [11] among them provide too small deuterium abundance to accept concordant $\Omega_{b}$ which satisfies the abundance constraints on the other light elements ${ }^{3} \mathrm{He},{ }^{4} \mathrm{He}$ and ${ }^{7} \mathrm{Li}$. The observed deuterium abundance still scatters largely by one order of magnitude depending on different Lyman- $\alpha$ systems, and there are still many error sources unclear in the analysis of abundance determination. However, if these detections 
are real, the abundance found there is presumed to constrain most strongly the primordial abundance because these clouds are the primitive gas which still resides in the epoch of galaxy formation and has not been processed very much in its evolutionary history.

It is the purpose of this paper to propose that the inhomogeneous cosmological model with degenerate electron-neutrino can resolve these two difficulties simultaneously within the framework of the standard model for neutrino. In the next section we first discuss neutrino properties in the early Universe which can affect strongly the primordial nucleosynthesis. We then present the results of primordial nucleosynthesis calculated in both homogeneous and inhomogeneous cosmological models in sect. 3 , and the $\Omega_{b}$ problem and the problem of overproduction of ${ }^{4} \mathrm{He}$ are discussed in details. Finally, in sect. 4 , we summarize this paper.

\section{Neutrino in the Early Universe}

Radiation and relativistic particles played more important role than ordinary matter in the evolution of early hot Big-Bang Universe. Since relativistic neutrinos had energy density comparable to the densities due to photons and charged leptons, a small modification of neutrino properties can change the expansion rate of the Universe and a resultant slight shift of weak decoupling temperature affects strongly the primordial nucleosynthesis [12].

Let us consider the ${ }^{4} \mathrm{He}$ synthesis in this section in order to make clear the role of neutrinos in primordial nucleosynthesis. Although all produced elements are, in principle, influenced by the weak interactions, sensitivity of inferred $\Omega_{b}$ to primordial ${ }^{4} \mathrm{He}$ abundance is very critical to resolve the crises for the following two reasons [1]. First, the neutronto-proton ratio before nucleosynthesis is delicately determined by the weak interactions between nucleons and leptons, which controls sensitively the ${ }^{4} \mathrm{He}$ yield. The second reason is that the ${ }^{4} \mathrm{He}$ abundance in HII regions is so accurately studied observationally that even one percent change in predicted abundance results in different $\Omega_{b}$ value. This is to be compared with less accurate abundance constraints on the other elements like D and ${ }^{7} \mathrm{Li}$ whose primordial abundances are only known by order.

\subsection{Neutron-to-Proton Ratio and Weak Decoupling}

The nucleosynthesis process can be studied quantitatively only by numerical calculation of solving a large number of non-linear rate equations because nuclear statistical equilibrium is not maintained down to $T \sim 100 \mathrm{keV}\left(\sim 10^{9} \mathrm{~K}\right)$ at which explosive nucleosynthesis occurs. However, since the major nuclear reactions are mediated by the strong and electromagnetic interactions, except for beta decays of triton, ${ }^{7} \mathrm{Be}$, etc., total numbers of protons and neutrons are approximately conserved. This avoids complications in our qualitatively discussion of ${ }^{4} \mathrm{He}$ synthesis in this section. The only effect on the neutronto-proton abundance ratio is the free decay of neutrons. Taking this into consideration, the ratio just before the onset of nucleosynthesis is given by

$$
\mathrm{r}(T) \equiv n_{\mathrm{n}} / n_{\mathrm{p}}=\mathrm{r}\left(T_{d}\right) \exp \left(-t / \tau_{n}\right),
$$

where $n_{\mathrm{n}}$ and $n_{\mathrm{p}}$ are the neutron and proton number densities, $\tau_{n}$ is the neutron mean life, and $\mathrm{r}\left(T_{d}\right)$ is the ratio at decoupling temperature of weak interactions.

Weak decoupling is defined at the time when the collision time scale of weak interactions, $\tau_{c} \sim<n_{\nu} \sigma v>^{-1}=G_{F}{ }^{-2} T^{-5}$, and the Hubble expansion time scale, $\tau_{H}=R / \dot{R}=$ 
$\left[1.66 T^{2} \sqrt{g^{*}} / M_{P l}\right]^{-1}$, balance with each other, where $G_{F}$ is Fermi coupling constant, $M_{P l}$ Planck mass, $g^{*}$ the effective degrees of freedom of relativistic particles to be discussed later, and $T$ the universal temperature. Equating these two time scales, we obtain

$$
T_{d} \sim\left[1.66 \sqrt{g^{*}} / M_{P l} / G_{F}^{2}\right]^{1 / 3} \sim O(1 \mathrm{MeV}) .
$$

At earlier times $t \lesssim 1 \mathrm{sec}$ and $T \gtrsim T_{d} \sim 1 \mathrm{MeV}, \tau_{c} \lesssim \tau_{H}$ holds and protons and neutrons are in chemical equilibrium by frequent weak interactions, $\mathrm{n}+\nu_{e} \leftrightarrow \mathrm{p}+\mathrm{e}^{-}, \mathrm{n}+\mathrm{e}^{+} \leftrightarrow$ $\mathrm{p}+\overline{\nu_{e}}$, and $\mathrm{n} \leftrightarrow \mathrm{p}+\mathrm{e}^{-}+\overline{\nu_{e}} \cdot r\left(T_{d}\right)$ is thus given by

$$
\mathrm{r}\left(T_{d}\right)=\left(m_{\mathrm{n}} / m_{\mathrm{p}}\right)^{3 / 2} \exp \left(-\delta m / T_{d}-\mu_{\nu_{e}} / T_{d}\right)
$$

where $\delta m=m_{\mathrm{n}}-m_{\mathrm{p}}=1.293 \mathrm{MeV}$ is the neutron-proton mass difference, and $\mu_{\nu_{e}}$ the chemical potential of electron-neutrino. Shortly after the weak decoupling, electrons and positrons have pair annihilated to reheat the Universe for entropy conservation. After this epoch the weak processes which interconvert neutrons and protons freeze out, and the abundance ratio $\mathrm{r}(T)$ stays at a value close to $\mathrm{r}\left(T_{d}\right)$.

Once nucleosynthesis occurs explosively, nuclear reactions incorporate almost all available neutrons into ${ }^{4} \mathrm{He}$, which has the highest binding energy among all light nuclei. The Coulomb barrier for the ${ }^{4} \mathrm{He}$-induced reactions with other charged nuclei prevents them from destroying easily once it is created. Thus, the mass fraction of ${ }^{4} \mathrm{He}$ is simply approximated by

$$
Y_{\mathrm{p}} \sim 2 n_{\mathrm{n}} /\left(n_{\mathrm{n}}+n_{\mathrm{p}}\right)=2 \mathrm{r}(T) /(1+\mathrm{r}(T))
$$

at the risk of losing quantitative accuracy which should follow the full network calculation to be discussed in sect. 3 .

\subsection{Partial Degenerate Neutrino}

An arbitrary conjecture which has been assumed in the standard cosmological model for primordial nucleosynthesis is that the net lepton number, $L_{x}=\left\{n_{\nu_{x}}-n_{\overline{\nu_{x}}}+n_{x}-n_{\bar{x}}\right\} / n_{\gamma}$ ( $x=e, \mu$ or $\tau$ ), is zero. However, any particle-physics and cosmological constraints do not rule out finite lepton asymmetry of the Universe within the framework of the standard model for quarks and leptons. It is even natural to assume $L_{x} \neq 0$ cosmologically.

After the epoch of cosmic quark-hadron phase transition which occurred at $T \sim 100$ $\mathrm{MeV}$, only abundant charged leptons were electrons and positrons. For charge neutrality, however, $\left(n_{e^{-}}-n_{e^{+}}\right) / n_{\gamma}=n_{B} / n_{\gamma} \leq 10^{-8}$ for the $\Omega_{b} \leq 1$ Universe models. Therefore, chemical potential of the electron must be negligibly small. Only neutrinos $\nu_{e}, \nu_{\mu}$ and $\nu_{\tau}$ and their antiparticles might have non-vanishing chemical potentials. Since electronlepton number and also muon- and tau-lepton numbers are believed to be conserved separately, the degeneracy parameters $\xi_{\nu_{x}}=\mu_{\nu_{x}} / k T$ must be constant.

Let us consider partial degeneracy of the only electron-neutrino, $\left|\xi_{\nu_{e}}\right| \ll 1$, in order to see the effects on ${ }^{4}$ He synthesis through eqs. (1) to (4). We are not interested in degeneracy of the other neutrino species because these are insensitive to primordial nucleosynthesis. (see the discussion below). We also discuss neither complete neutrino degeneracy which has been studied in literature. 
There are two different effects of partial electron-neutrino degeneracy on nucleosynthesis. The first effect arises from additional energy density due to degenerate neutrinos:

$$
\left(\rho_{\nu_{e}}+\rho_{\overline{\nu_{e}}}\right) / \rho_{\gamma}=\left(T_{\nu_{e}} / T_{\gamma}\right)^{4}\left[7 / 8+15 / 4 \pi^{2}{\xi_{\nu_{e}}}^{2}+15 / 8 \pi^{4} \xi_{\nu_{e}}^{4}\right] .
$$

In the limit of vanishing chemical potentials for all relativistic particles, the effective degrees of freedom $g^{*}$ which appeared in eq. (2) is given by $g^{*}=\sum_{b=\text { Bosons }} g_{b}+(7 / 8) \sum_{f=\text { Fermions }} g_{f}$. The second and third terms of r.h.s. in eq. (5) are additional degrees of freedom for degenerate neutrinos, $\Delta g^{*}$. Combining eqs. (1) - (4) with this change from $g^{*}$ to $g^{*}+\Delta g^{*}$ and artificially suppressing the change in neutron-to-proton ratio due to $\exp \left(-\mu_{\nu_{e}} / T\right)$ in eq. (3), we can estimate the change in the mass fraction of ${ }^{4} \mathrm{He}$ :

$$
\Delta Y_{\mathrm{p}} \sim 1 / 6 Y_{\mathrm{p}}\left(1-Y_{\mathrm{p}} / 2\right)\left(\delta m / T_{d}\right) \Delta g^{*} / g^{*}>0 .
$$

This is a second order effect of $\xi_{\nu_{e}}$, i.e. $\Delta g^{*} \sim \xi_{\nu_{e}}{ }^{2}$.

The second more important effect arises directly from the factor $\exp \left(-\mu_{\nu_{e}} / T\right)$ which we ignored artificially in the above discussion. A simple calculation leads to the change in $Y_{\mathrm{p}}$

$$
\Delta Y_{\mathrm{p}} \sim-Y_{\mathrm{p}}\left(1-Y_{\mathrm{p}} / 2\right) \xi_{\nu_{e}}<0
$$

which is a first order effect of $\xi_{\nu_{e}}$. Having calculated these two effects (6) and (7), we can summarize qualitatively that the net ${ }^{4} \mathrm{He}$ abundance decreases if electron-neutrino are partially degenerate with positive $\xi_{\nu_{e}}$.

\section{Numerical Result of Primordial Nucleosynthesis}

Important result of the last section is that ${ }^{4} \mathrm{He}$ abundance is reduced for partial electronneutrino degeneracy with arbitrary $\Omega_{b}$. Since ${ }^{4} \mathrm{He}$ abundance increases monotonically with increasing $\Omega_{b}$ as shown in Figure 1 1 , allowed $\Omega_{b}$ can be larger for satisfying the same ${ }^{4} \mathrm{He}$ abundance constraint. Let us quantify this by fulfilling the full network calculation of primordial nucleosynthesis.

\subsection{Homogeneous Model}

Shown in Figure 1 1 is the comparison between the observed abundance constraints on ${ }^{4} \mathrm{He}$ and $\mathrm{D}$ and the calculated curve in the homogeneous Big-Bang model as a function of $\eta$ for various $\xi_{\nu_{e}}$, where $\eta$ is the baryon-to-photon ratio, $\eta=n_{B} / n_{\gamma}$, and is linearly proportional to $\Omega_{b}, \Omega_{b}=1.464 \times 10^{8} \eta h_{50}^{-2}$. Calculated abundance curves with $\xi_{\nu_{e}}=0$ cannot find concordant $\Omega_{b}$ to satisfy both ${ }^{4} \mathrm{He}$ and D. Note that mass fraction of ${ }^{4} \mathrm{He}$, $Y_{\mathrm{p}}$, is displayed in linear scale, while the deuterium abundance relative to hydrogen, $\mathrm{D} / \mathrm{H}$ $=n_{\mathrm{D}} / n_{\mathrm{H}}$, is shown in logarithmic scale. Although observed deuterium abundance from many different samples scatters largely, the constraint on ${ }^{4} \mathrm{He}, 0.226 \leq Y_{\mathrm{p}} \leq 0.247$, is very accurate. This fact helps determine the most likely $\xi_{\nu_{e}}$. The homogeneous model with $\xi_{\nu_{e}} \sim 0.05$ can best fit ${ }^{4} \mathrm{He}$ abundance as well as low deuterium abundance $\mathrm{D} / \mathrm{H} \sim 10^{-5}$.

Unfortunately, however, the concordant $\Omega_{b}$ does not reach the observed value detected

as hot X-ray gas, $0.1 \leq \Omega_{b} h_{50}^{3 / 2} \leq 0.3$, in rich clusters of galaxies. Figure 2(a) shows dependence of both theoretically and observationally inferred $\Omega_{b}$ on the Hubble constant. The homogeneous model fails in explaining observed large $\Omega_{b}$ value in reasonable range of the Hubble constant $0.5 \leq h_{50} \leq 1.0$. 


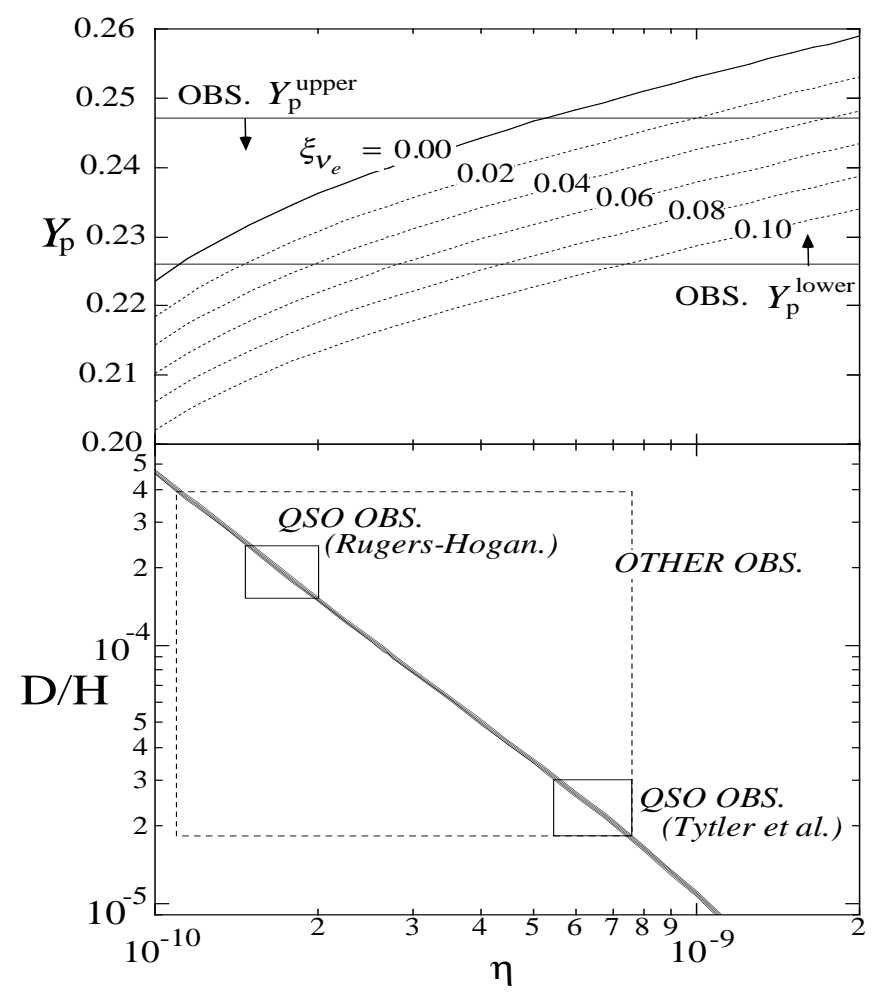

Figure 1. $Y_{\mathrm{p}}$ and $\mathrm{D} / \mathrm{H}$ versus $\eta$ for various degeneracy parameters of electron-neutrino $\xi_{\nu_{e}}$ in the homogeneous Big-Bang model. Observed abundance constraints on $\mathrm{D} / \mathrm{H}$ are from Rugers-Hogan [10], Tytler et al. [11], and references therein.

\subsection{Inhomogeneous Model}

It was found in our recent studies [8,9] that the inhomogeneous Big-Bang model, which assumes non-degenerate neutrino, allows larger $\Omega_{b}$. If the inhomogeneous baryon-density distribution is created in some elementary process like cosmic QCD phase transition and others and if the scale of density inhomogeneities is comparable to the diffusion length of neutrons at the epoch of primordial nucleosynthesis, then $\Omega_{b}$ dependence of primordial nucleosynthesis is very different from the homogeneous model [5]-[0]. Neutrons can easily diffuse out of the fluctuations for charge neutrality, while charged nuclei stay almost inside the fluctuations, thus forming specific density distributions. In high-density proton rich region is the neutron-to-proton ratio even smaller due to prominent neutron diffusion. ${ }^{4} \mathrm{He}$ production is suppressed here. On the other hand, ${ }^{4} \mathrm{He}$ production is regulated by small amount of protons. Thus the net ${ }^{4} \mathrm{He}$ production is reduced in the inhomogeneous model, though both diffusion and back-diffusion of all nuclides including neutrons make the process more complicated [8.99].

The diffusion of all nuclides including neutrons starts operating when electrons and positrons pair annihilate shortly after the weak decoupling and interconversion between 

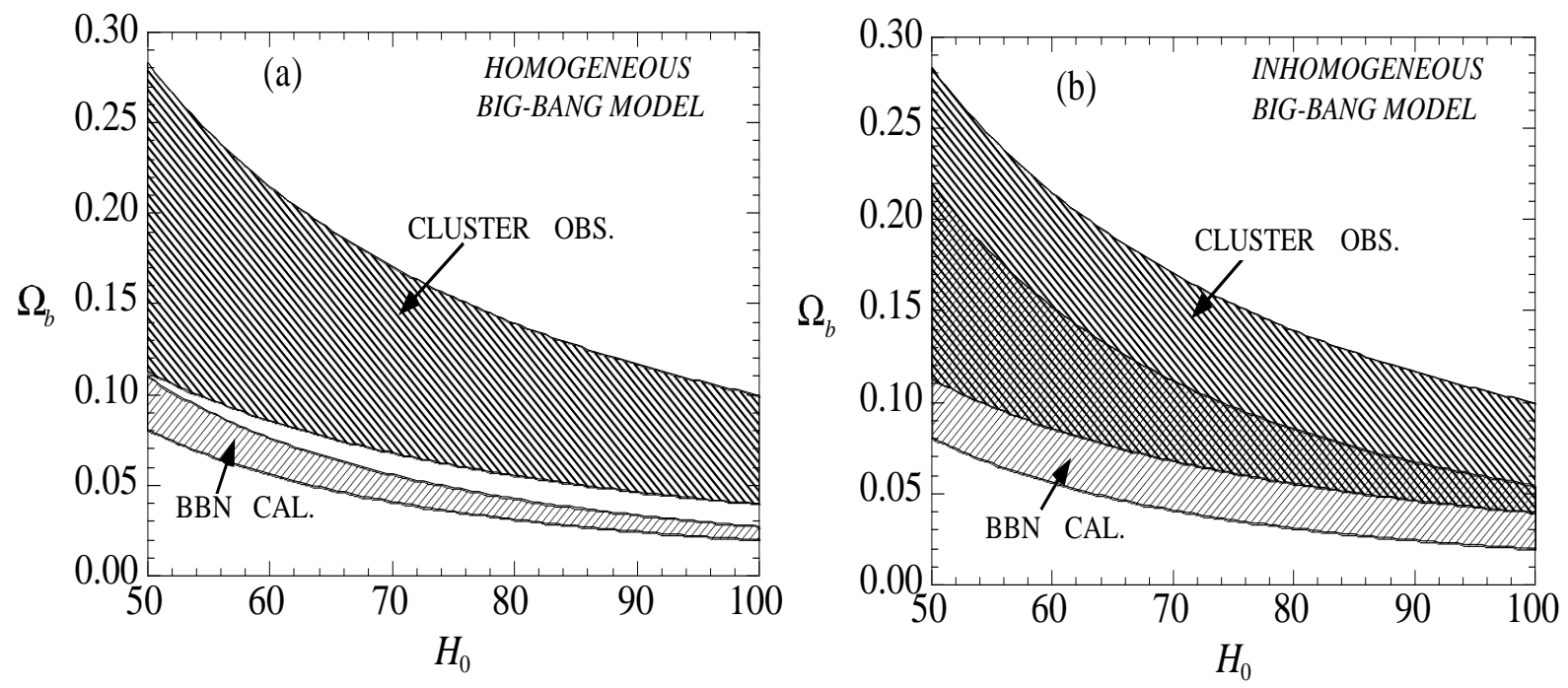

Figure 2. Allowed $\Omega_{b}$ value versus $H_{0}$, inferred from astronomical observations of rich clusters of galaxies (CLUSTER OBS) [3] and from the present theoretical calculations in (a) homogeneous and (b) inhomogeneous Big-Bang models (BBN CAL).

protons and neutrons stops. Degeneracy of electron-neutrino determines the neutron-toproton ratio at this epoch before efficient diffusion starts. Therefore, nucleosynthesis is affected by the neutrino degeneracy in the inhomogeneous Big-Bang model as well.

The primordial nucleosynthesis calculated in the inhomogeneous model with $\xi_{\nu_{e}}=0.0$ and 0.05 are displayed in Figure 3 where contour map of each light element abundance is shown in the $r-\Omega_{b}(r-\eta)$ plane, where $r$ is the mean separation radius between fluctuating high-density regions at the $T=1 \mathrm{MeV}$ epoch. For the same mechanism as in the homogeneous model, ${ }^{4} \mathrm{He}$ abundance pattern changes drastically, while the other elements do not change very much. Only when $\xi_{\nu_{e}}=0.05$ is adopted, $\Omega_{b} h_{50}^{2}$ as large as 0.22 is allowed for $r \sim 10^{4}$ meter (at $T=1 \mathrm{MeV}$ ), even satisfying the light element abundance constraints whose detail is reported elsewhere. This allowed value of $\Omega_{b}$ is now in reasonable agreement with astronomical observation of hot X-ray gas, $0.1 \leq \Omega_{b} h_{50}^{3 / 2} \leq$ 0.3, as clearly shown in Figure 2(b).

\section{Conclusion}

We studied the effects of lepton asymmetry of partially degenerate electron-neutrino on the primordial nucleosynthesis. Homogeneous Big-Bang model with neutrino degeneracy parameter $\xi_{\nu_{e}}=0.05$ can recover the concordance between ${ }^{4} \mathrm{He}$ and low deuterium abundance which was found in some Lyman- $\alpha$ clouds along the line of sight to high red-shift quasars, but the resultant $\Omega_{b}$ is less than detected in rich clusters $0.1 \leq \Omega_{b} h_{50}^{3 / 2} \leq 0.3$. It was found that the inhomogeneous Big-Bang model with the same degeneracy parameter can predict $\Omega_{b}$ as large as 0.22 , which is in reasonable agreement with observation. This degeneracy parameter corresponds to a small chemical potential of electron-neutrino of order $10^{-5} \mathrm{eV}$. It is desirable to detect the asymmetry of background neutrinos. 


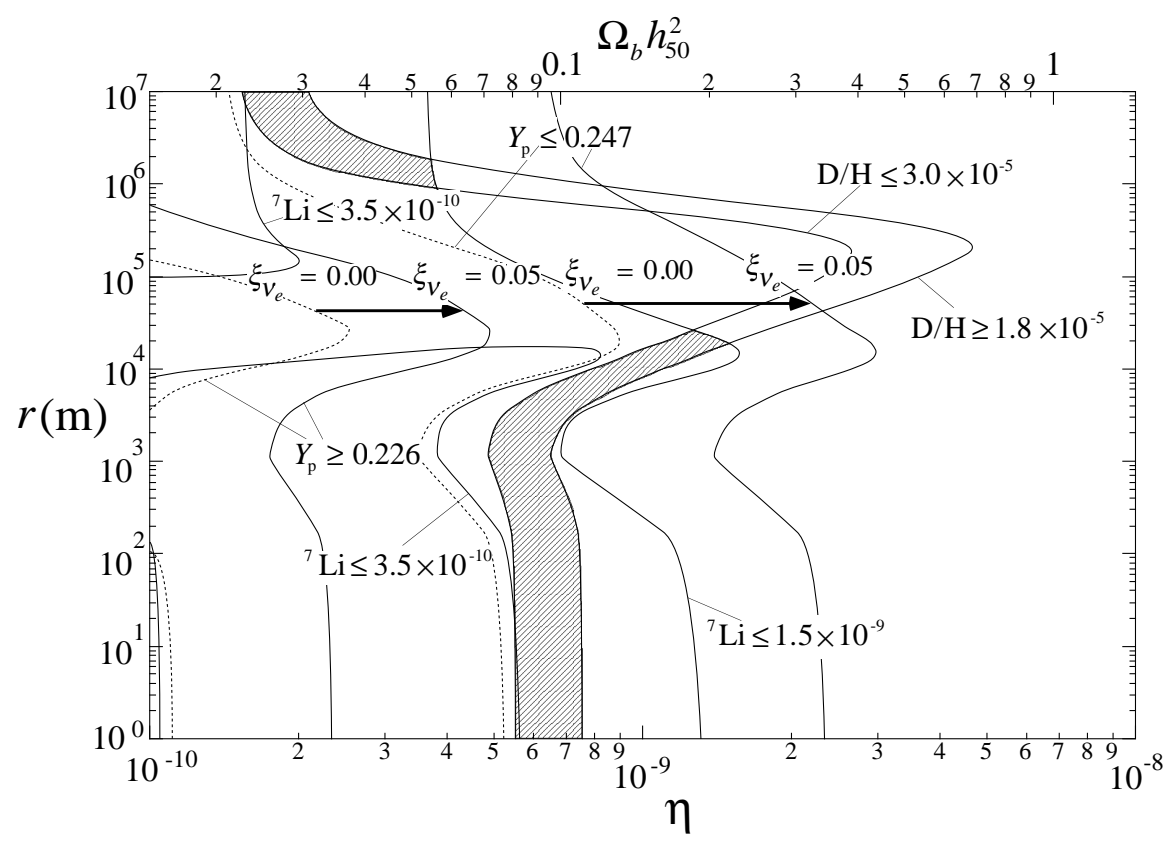

Figure 3. Contours of allowed values for $\eta$ or $\left(\Omega_{b} h_{50}^{2}\right)$ and fluctuation separation radius $r$ (at $T=1 \mathrm{MeV}$ ). This calculation is based upon baryon density fluctuations represented by cylindrical shells (see ref. [8] for details). The hatched region satisfies the light-element abundance constraints, as indicated, for degeneracy parameter of electron-neutrino $\xi_{\nu_{e}}=$ 0.05 .

\section{REFERENCES}

1. R. V. Wagoner, W. A. Fowler, \& F. Hoyle, ApJ. 148 (1967) 3

2. C. J. Copi, D. N. Schramm, \& M. S. Turner, Science. 267 (1995) 192, and reference therein.

3. S. D. M. White, J. F. Navarro, A. E. Evrard, \& C. S. Frenk, Nature. 366 (1993) 429

4. N. A. Bahcall, L. M. Lubin, \& V. Dorman, ApJ. 447 (1995) L81

5. J. H. Applegate, \& C. J. Hogan, Phys. Rev. D. 30 (1985) 3037

6. C. R. Alcock, G. M. Fuller, \& G. J. Mathews, ApJ. 320 (1987) 439

7. T. Kajino \& R. N. Boyd, ApJ. 359 (1990) 267

8. M. Orito, T. Kajino, R. N. Boyd, \& G. J. Mathews, ApJ. 488 (1997)

9. G. J. Mathews, T. Kajino, \& M. Orito, ApJ. 456 (1996) 98

10. M. Rugers, \& C. J. Hogan, ApJ. 459 (1996) L1

11. D. Tytler, X. M. Fan, \& S. Burles, Nature. 381 (1996) 207

12. S. Weinberg, Gravitation and Cosmology, John Wiley and Sons ed. (1972) 